\section{Clinical Evaluation of Measurement Method for Antigen Specific IgE in Tears of Patients with Allergic Conjunctival Disease}

Purpose: To investigate a method for determining antigen specific IgE antibodies in tears of patients with allergic keratoconjunctival disease.

Subjects and Methods: Antigen specific IgE antibodies to Japanese cedar pollen or housedust-mites in tears of patients with allergic conjunctival diseases were examined. Both eyes in each patient were examined. The right eye was examined in normal healthy volunteers as a control. The number of eyes examined for $\operatorname{IgE}$ antibodies to Japanese cedar pollen was 32 eyes with seasonal allergic conjunctivitis (SAC), 12 eyes with perennial allergic conjunctivitis (PAC), 12 eyes with atopic keratoconjunctivitis (AKC), 4 eyes with vernal keratoconjunctivitis (VKC), and 12 eyes of the controls. The number of eyes examined for IgE antibodies to housedust-mites was 32 eyes with SAC, 20 eyes with PAC, 30 eyes with AKC, 22 eyes with VKC, and 10 eyes of the controls. Tears were sampled by the method of Schirmer Test-1. Sampled tears were eluted with $200 \mu$ of phosphate buffered solution $(\mathrm{pH} 7.2,0.05 \mathrm{M})$ and analyzed by the AlaSTAT-IMMULYZE ${ }^{\circledR}$ method. Recovery rate of absorbance by filter paper for $\mathrm{IgE}$ antibodies in tears was determined by ratio to the standard sample of serum solution containing housedust-mite specific IgE antibodies with known concentration.

Results: Recovery rate from filter paper of $\mathrm{IgE}$ antibodies in tears was $83.6 \%$. IgE antibodies to Japanese cedar pollen were detected in the tears of 11 of 32 eyes with SAC. In other subjects, IgE antibodies were under the limit of the detection concentration except one eye of a patient with $\mathrm{AKC}$. In the positive cases of Japanese cedar pollen specific IgE antibodies, there was no significant difference in the concentration between the right and the left eyes. IgE antibodies to housedust-mites were significantly higher not only in incidence but also in concentration in 18 of 22 eyes with $\mathrm{VKC}$ than in other allergic conjunctival disease groups. IgE antibodies were also detected in 8 of 20 eyes with PAC and 12 of 30 eyes with AKC.

Conclusion: The method for determining antigen specific $\operatorname{IgE}$ antibodies in tears is clinically useful to diagnose and to investigate the pathophysiology of allergic conjunctival diseases.

Nippon Ganka Gakkai Zasshi (J Jpn Ophthalmol Soc 107:578-582, 2003)

\author{
Minoru Kitazawa ${ }^{1}$, Jun Shoji ${ }^{1}$, Noriko Inada ${ }^{1}$, Mitsuru Sawa ${ }^{1}$ and \\ Hiroshi Kato ${ }^{2}$ \\ ${ }^{1}$ Department of Ophthalmology, Nihon University School of \\ Medicine; ${ }^{2}$ Iatron Laboratories, Inc.
}

DOI 10.1007/s10384-004-1018-2

\section{Glutamate and $\gamma$-aminobutyric Acid Release into the Vitreous During Cataract Surgery}

Objective: In cataract surgery, transient retinal ischemia and photostimulation-induced retinal damage occurs, even though only for a short duration. We suspected the possibility of excessive release of glutamate under these conditions, and therefore analyzed the amino acid contents in the vitreous body.

Materials and Methods: The vitreous bodies of 32 eyes with disease of the macular area necessitating retinovitreous surgery were used as samples for amino acid analysis. Ischemic diseases such as diabetic retinopathy, venous occlusion, and retinal detachment were excluded. The vitreous amino acid contents were compared between 18 eyes that underwent combined cataract and vitreous surgery and 14 eyes that had vitreous surgery alone.

Results: An approximately 3-fold increase of glutamate, 6fold increase of $\gamma$-aminobutyric acid (GABA), and 2-fold increase of alanine were observed in eyes undergoing combined cataract and vitreous surgery compared to vitreous surgery alone.

Conclusion: Even in human eyes, acute pressure overload or photostimulation for a short duration causes excessive release of glutamate. A simultaneous increase of GABA suggests the possibility that when glutamate is overreleased, the retina physiologically reduces the concentration of glutamate via GABA, in an attempt to suppress the neurotoxin action.

Nippon Ganka Gakkai Zasshi (J Jpn Ophthalmol Soc 107:583-589, 2003)

Jun Kezuka, Yoshihiro Wakabayashi, Tomoyuki Yagihashi, Takuya Iwasaki and Masahiko Usui

Department of Ophthalmology, Tokyo Medical University

DOI 10.1007/s10384-004-1019-1

\section{Vitrectomy for Endophthalmitis after Cataract Surgery}

Purpose: To identify risk factors of poor visual outcome with vitrectomy for early-onset endophthalmitis after cataract surgery.

Patients and Methods: Clinical records of 29 consecutive eyes with endophthalmitis developing within 6 weeks after cataract surgery and that underwent therapeutic vitrectomy between June 1996 and April 2001 were retrospectively reviewed. Twenty-two of the eyes received intravitreal injections of vancomycin and ceftazidime at the time of vitrectomy, and all patients received intravenous antibiotics. Eyes were divided into two groups; group A consisted of 22 eyes with a final visual acuity of 0.2 or greater, and group B consisted of 7 eyes with a final visual acuity of less than 0.2 . 